\title{
SEIBERG-WITTEN INVARIANTS AND (ANTI-)SYMPLECTIC INVOLUTIONS
}

\author{
YONG SEUNG CHO and YOON HI HONG \\ Department of Mathematics, Ewha Women's University, Seoul 120-750, Korea \\ e-mail:yescho@ewha.ac.kr,yoonihong@hanmail.net
}

(Received 16 November, 2000; accepted 18 February, 2003)

\begin{abstract}
Let $X$ be a closed, symplectic 4-manifold. Suppose that there is either a symplectic or an anti-symplectic involution $\sigma: X \rightarrow X$ with a 2-dimensional compact, oriented submanifold $\Sigma$ as a fixed point set.

If $\sigma$ is a symplectic involution then the quotient $X / \sigma$ with $b_{2}^{+}(X / \sigma) \geq 1$ is a symplectic 4-manifold.

If $\sigma$ is an anti-symplectic involution and $\Sigma$ has genus greater than 1 representing non-trivial homology class, we prove a vanishing theorem on Seiberg-Witten invariants of the quotient $X / \sigma$ with $b_{2}^{+}(X / \sigma)>1$.

If $\Sigma$ is a torus with self-intersection number 0 , we get a relation between the Seiberg-Witten invariants on $X$ and those of $X / \sigma$ with $b_{2}^{+}(X), b_{2}^{+}(X / \sigma)>2$ which was obtained in [21] when the genus $g(\Sigma)>1$ and $\Sigma \cdot \Sigma=0$.

2000 Mathematics Subject Classification. 53D05, 57M12, 57M50, 57R17, 57R57, $57 \mathrm{~S} 25$.
\end{abstract}

1. Introduction. Let $X$ be a closed, oriented Riemannian 4-manifold and let $L \rightarrow X$ be a complex line bundle satisfying $c_{1}(L)=w_{2}(T X) \bmod 2$. Then there is a principal $\operatorname{Spin}^{c}(4)$-bundle $\xi \rightarrow X$ associated to $L$. We say $\xi$ is a $\operatorname{Spin}^{c}$-structure associated to $L$. Let $W^{ \pm}(\xi)$ be $\left( \pm \frac{1}{2}\right)$-twisted spinor bundles associated to $\xi$. The determinant bundle det $W^{ \pm}$is isomorphic to $L$.

Let $\mathcal{A}(L)$ be the set of all Riemannian connections on $L$. The gauge group $\mathcal{G}(L)$ of all bundle automorphisms on $L$ acts on $\mathcal{A}(L) \times \Gamma\left(W^{+}(\xi)\right)$ by $g(A, \psi)=$ $\left(A-2 g^{-1} d g, g \psi\right)$, for all $g \in \mathcal{G}(L)$ and $(A, \psi) \in \mathcal{A}(L) \times \Gamma\left(W^{+}(\xi)\right)$.

For a unitary connection $A \in \mathcal{A}(L)$ and a positive spinor field $\psi \in \Gamma\left(W^{+}(\xi)\right)$ the Seiberg-Witten equations are defined by

$$
\left\{\begin{array}{l}
F_{A}^{+}=q(\psi), \\
D_{A} \psi=0,
\end{array}\right.
$$

where $D_{A}: \Gamma\left(W^{+}(\xi)\right) \rightarrow \Gamma\left(W^{-}(\xi)\right)$ is the Dirac operator associated with $A$ and $q$ : $C^{\infty}\left(W^{+}(\xi)\right) \rightarrow \Omega_{X}^{+}(i \mathbb{R})$ is a quadratic map defined by $q(\psi)=\psi \otimes \psi^{*}-\frac{\|\psi\|^{2}}{2} \mathrm{Id}$.

Let $\mathcal{M}(\xi)$ be the moduli space of the gauge equivalence classes of all solutions of the Seiberg-Witten equations. Then $\mathcal{M}(\xi)$ is compact by [16].

This work was supported by a Korea Research Foundation Grant (No KRF-2002-072-C00010). 
We consider perturbed Seiberg-Witten equations:

$$
\left\{\begin{array}{l}
F_{A}^{+}+i \delta=q(\psi), \\
D_{A} \psi=0,
\end{array}\right.
$$

where $\delta$ is a real valued, self-dual 2-form on $X$.

Then the perturbed moduli space $\mathcal{M}_{\delta}(\xi)$ is a smooth manifold with its dimension $\operatorname{dim} \mathcal{M}_{\delta}(\xi)=\frac{1}{4}\left\{c_{1}(L)^{2}[X]-(2 \chi(X)+3 \operatorname{Sign}(X))\right\}$. If the metric on $X$ is chosen so that the perturbed Seiberg-Witten equations admit no reducible solutions, then $\mathcal{M}_{\delta}(\xi)$ is compact. If $\operatorname{dim} \mathcal{M}_{\delta}(\xi)=2 d$, then the Seiberg-Witten invariant is defined by

$$
\int_{\mathcal{M}_{\delta}(\xi)} c_{1}\left(\mathcal{M}_{\delta}(\xi)_{0}\right)^{d}
$$

the integral of the maximal power of the Chern class of the circle bundle $\mathcal{M}_{\delta}(\xi)_{0} \rightarrow \mathcal{M}_{\delta}(\xi)$, where $\mathcal{M}_{\delta}(\xi)_{0}$ is the framed moduli space.

If $\operatorname{dim} \mathcal{M}_{\delta}(\xi)$ is odd or negative then the Seiberg-Witten invariant is defined to be zero. For details, see [23].

In general, there are infinitely many elements $c_{1}(L) \in H^{2}(X ; \mathbb{Z})$ satisfying $c_{1}(L)=w_{2}(T X) \bmod 2$. Each such element induces a $\operatorname{Spin}^{c}$-structure on $X$. However there are only finitely many elements in $H^{2}(X ; \mathbb{Z})$ such that their Seiberg-Witten invariants are non-zero. Such an element in $H^{2}(X ; \mathbb{Z})$ is called a basic class. Hence the set of basic classes is finite. Furthermore $X$ is said to have simple type if all basic classes satisfy $c_{1}(L)^{2}[X]=2 \chi(X)+3 \sigma(X)$.

Using the Seiberg-Witten invariants, Taubes [23] proved the non-trivialness of the Seiberg-Witten invariants on symplectic 4-manifolds with $b_{2}^{+}>1$. In Section 2, we consider a symplectic involution $\sigma$ over a closed symplectic 4-manifold $X$ with a symplectic structure $\omega$. We show that if the symplectic involution $\sigma$ has a 2dimensional, compact, oriented submanifold as a fixed point set, then the quotient $X / \sigma$ with $b_{2}^{+}(X / \sigma) \geq 1$ is a closed symplectic 4-manifold.

If a closed, oriented Riemannian 4-manifold $X$ has a basic class, it gives a minimal genus bound for the embedded surface, called the adjunction inequality.

THEOREM 1.1. (Adjunction Inequality $[\mathbf{1 6 , 1 9 ]}$ ). Let $X$ be a smooth 4-manifold with $b_{2}^{+}(X)>1$ and a basic class $L$, and let $\Sigma_{X}$ be an embedded connected oriented surface with $\Sigma_{X} \cdot \Sigma_{X} \geq 0$ and $\left[\Sigma_{X}\right] \neq 0 \in H_{2}(X ; \mathbb{Z})$. Then we have an inequality

$$
-\chi\left(\Sigma_{X}\right) \geq \Sigma_{X} \cdot \Sigma_{X}+\left|c_{1}(L)\left[\Sigma_{X}\right]\right| .
$$

Ozsváth and Szabó [20] extended Theorem 1.1 for a 4-manifold $X$ of SeibergWitten simple type with $b_{2}^{+}(X)>1$ and $g\left(\Sigma_{X}\right)>0$ and $\Sigma_{X} \cdot \Sigma_{X}<0$.

Related with a symplectic 4-manifold, there is a Akbulut's conjecture [15] (Problem 4.104) that if an anti-symplectic involution $\sigma$ acts on a simply-connected, closed, symplectic 4-manifold $X$ (that is, $\sigma^{*} \omega=-\omega$ for a symplectic structure $\omega$ ) with a 2dimensional smooth surface as a fixed point set, then $X / \sigma=r \mathbb{C} P^{2} \sharp s \mathbb{C} \bar{P}^{2}$ or $n S^{2} \times S^{2}$, for some $r, s, n, \in \mathbb{N}$.

Akbulut [1] showed that if $\sigma$ is a complex conjugation over a complex algebraic surface $X$ with a real algebraic surface as a fixed point set then $X / \sigma=r \mathbb{C} P^{2} \sharp s \mathbb{C} \bar{P}^{2}$ or $n S^{2} \times S^{2}$ for many cases.

In Section 3, we consider an anti-symplectic involution $\sigma$ over a symplectic 4manifold $X$ with a 2-dimensional compact submanifold as a fixed point set. By using 
Theorem 1.1, we show that if the fixed point set contains a Riemann surface with genus greater than 1 representing non trivial homology class in $H_{2}(X / \sigma ; \mathbb{Z})$, then the quotient $X / \sigma$ with $b_{2}^{+}(X / \sigma)>1$ has a vanishing Seiberg-Witten invariant.

Let $X^{\prime}$ be a closed, smooth, oriented 4-manifold with a smoothly embedded 2torus $T^{2}$ with self-intersection number 0 and let $\pi: X \rightarrow X^{\prime}$ be a double cover branched along $T^{2}$. In Section 4, we prove a relation between the Seiberg-Witten invariants on $X$ and those of $X^{\prime}$ when $b_{2}^{+}(X), b_{2}^{+}\left(X^{\prime}\right)>2$. Ruan and Wang [21] proved the same results when the genus of the fixed point set is greater than 1 and the fixed point set has selfintersection number 0 . In particular, if $\sigma: X \rightarrow X$ is an anti-symplectic involution on a closed symplectic 4-manifold $X$ whose fixed point set is a torus with self-intersection number 0 , we get a relation between the Seiberg-Witten invariants on $X$ and those of $X / \sigma$.

In Section 5, we calculate Theorem 4.7 for some cases.

2. Seiberg-Witten invariant of the quotient manifold under a symplectic involution with a 2-dimensional fixed point set. Let $X$ be a closed symplectic 4-manifold with a symplectic structure $\omega$. A smooth map $\sigma: X \rightarrow X$ is a symplectic involution if and only if $\sigma^{*} \omega=\omega$ and $\sigma^{2}=\operatorname{Id}$ on $X$.

Proposition 2.1. Let $X$ be a closed symplectic 4-manifold with a symplectic structure $\omega$. Suppose that $\sigma: X \rightarrow X$ is a symplectic involution with a 2-dimensional, compact, oriented submanifold $\Sigma$. Then $\Sigma$ is a symplectic submanifold.

Proof. By definition, $J$ is a $\omega$-compatible almost complex structure if and only if $\omega(v, J v)>0$, for all $v \neq 0 \in T X$, and $\omega(J v, J w)=\omega(v, w)$, for all $v, w \in T X$. It is known that the set of all $\omega$-compatible almost complex structures is not empty and contractible. Then we can find a $\omega$-compatible metric $g$ such that $g(v, w)=\omega(v, J w)$ and $\omega$ is self-dual with respect to $g$.

Let $T \Sigma$ and $N_{\Sigma}$ be, respectively, the tangent and normal complex line bundles of $\Sigma$ in $X$. The induced map $\sigma_{*}$ on $\left.T X\right|_{\Sigma}=T \Sigma \oplus N_{\Sigma}$ satisfies $\left.\sigma_{*}\right|_{T \Sigma}=$ Id and $\left.\sigma_{*}\right|_{N_{\Sigma}}=-$ Id.

Then $\sigma$ acts as an isometry over $\left.T X\right|_{\Sigma}$ for the $\omega$-compatible metric $g$. Indeed, for all $v_{1}, v_{2} \in T \Sigma$ and $w_{1}, w_{2} \in N_{\Sigma}, g\left(v_{i}, w_{j}\right)=0, i, j=1,2$, and

$$
\begin{aligned}
\sigma^{*} g\left(v_{1}, v_{2}\right) & =g\left(\sigma_{*} v_{1}, \sigma_{*} v_{2}\right)=g\left(v_{1}, v_{2}\right), \\
\sigma^{*} g\left(w_{1}, w_{2}\right) & =g\left(\sigma_{*} w_{1}, \sigma_{*} w_{2}\right)=g\left(-w_{1},-w_{2}\right)=g\left(w_{1}, w_{2}\right) .
\end{aligned}
$$

Then we have

$$
\begin{gathered}
g\left(J \sigma_{*} v, w\right)=\omega\left(\sigma_{*} v, w\right)=\sigma^{*} \omega\left(\sigma_{*} v, w\right)=\omega\left(v, \sigma_{*} w\right)=g\left(J v, \sigma_{*} w\right) \\
=\sigma^{*} g\left(J v, \sigma_{*} w\right)=g\left(\sigma_{*} J v, w\right), \quad \text { for all } v,\left.w \in T X\right|_{\Sigma} .
\end{gathered}
$$

Thus $g\left(J \sigma_{*} v, w\right)=g\left(\sigma_{*} J v, w\right)$, for all $v,\left.w \in T X\right|_{\Sigma}$, and $J \circ \sigma_{*}=\sigma_{*} \circ J$ on $\left.T X\right|_{\Sigma}$. Then for all $v \in T \Sigma, J v \in T \Sigma$ and so $T \Sigma$ is a complex vector space.

For any non zero $v \in T \Sigma$, we have $J v \in T \Sigma$ and $\omega(v, J v)=g(v, v)>0$. Thus the restriction of $\omega$ on $\Sigma$ is a symplectic structure on $\Sigma$.

Proposition 2.2. Let $X$ be a closed symplectic 4-manifold with a symplectic structure $\omega$. Suppose that $\sigma: X \rightarrow X$ is a symplectic involution with a 2-dimensional compact, oriented submanifold $\Sigma$ as a fixed point set. Then the quotient $X / \sigma$ with $b_{2}^{+}(X / \sigma) \geq 1$ is a closed symplectic 4-manifold. 
Proof. Let $\pi: X \rightarrow X / \sigma$ be the projection and the image of the fixed point set $\pi(\Sigma)=\Sigma^{\prime}$. Then by [24] and [5], the quotient $X / \sigma$ is a closed, smooth 4-manifold.

By [4], there is a $\sigma$-invariant tubular neighborhood $N(\Sigma)$ of $\Sigma$ in $X$ such that the restriction $\left.\pi\right|_{N(\Sigma)}: N(\Sigma) \rightarrow \pi(N(\Sigma))=N\left(\Sigma^{\prime}\right)$ is a double covering with the branch set $\Sigma^{\prime}$ that is locally $\pi(z, v)=\pi(z,-v)=[z, v]$, for all $z \in \Sigma$ and $v$ in the normal fiber.

Since $\sigma^{*} \omega=\omega$, the $\sigma$-invariant symplectic form $\omega$ on $X$ defines naturally a symplectic form $\omega^{\prime}$ on $X / \sigma$ by $\omega^{\prime}\left(v^{\prime}, w^{\prime}\right)=\omega(v, w)$ if $\pi_{*}(v)=v^{\prime}, \pi_{*}(w)=w^{\prime}$ for all $v, w \in T X$.

Indeed, for all $x^{\prime} \in \Sigma^{\prime} \subset X / \sigma$, the tangent space $T_{x^{\prime}} X^{\prime}=\left.T_{x^{\prime}} \Sigma^{\prime} \oplus N_{\Sigma^{\prime}}\right|_{x^{\prime}}$ and locally $\omega^{\prime}=d x_{1}^{\prime} d x_{2}^{\prime}+d x_{3}^{\prime} d x_{4}^{\prime}$, where $x^{\prime}=\left(x_{1}^{\prime}, x_{2}^{\prime}\right)$ and $\left(x_{3}^{\prime}, x_{4}^{\prime}\right)$ is a coordinate of the normal fiber. Then there is an element $x \in \Sigma \subset X$ such that $\pi(x)=x^{\prime}, T_{x} X=\left.T_{x} \Sigma \oplus N_{\Sigma}\right|_{x}$ and locally $\omega=d x_{1} d x_{2}+d x_{3} d x_{4}$.

Let $v=\left(v_{1}, v_{2}\right), w=\left(w_{1}, w_{2}\right) \in T_{x} X=\left.T_{x} \Sigma \oplus N_{\Sigma}\right|_{x}$ and $\pi_{*} v=v^{\prime}, \pi_{*} w=w^{\prime}$. Then $\sigma_{*}\left(v_{1}, v_{2}\right)=\left(v_{1},-v_{2}\right)$ and we have

$$
\begin{gathered}
\omega(v, w)=\left(d x_{1} d x_{2}+d x_{3} d x_{4}\right)(v, w)=d x_{1} d x_{2}\left(v_{1}, w_{1}\right)+d x_{3} d x_{4}\left(v_{2}, w_{2}\right), \\
\sigma^{*} \omega(v, w)=\omega\left(\sigma_{*} v, \sigma_{*} w\right)=\omega\left(\left(v_{1},-v_{2}\right),\right. \\
\left.\left(w_{1},-w_{2}\right)\right)=d x_{1} d x_{2}\left(v_{1}, w_{1}\right)+d x_{3} d x_{4}\left(-v_{2},-w_{2}\right)=d x_{1} d x_{2}\left(v_{1}, w_{1}\right)+d x_{3} d x_{4}\left(v_{2}, w_{2}\right) .
\end{gathered}
$$

Thus $\omega^{\prime}$ is well-defined on $\Sigma^{\prime} \subset X / \sigma$. The other case $x \in X / \sigma-\Sigma^{\prime}$ is clear since $\sigma^{*} \omega=\omega$. Thus we have completed the proof.

EXAMPLE 2.3. [7]. Let $X=S^{2} \times S^{2}$ be the symplectic 4-manifold with the standard product symplectic form $\omega=\omega_{1}+\omega_{2}$, where $\omega_{1}$ and $\omega_{2}$ are the standard symplectic forms on $S^{2}$.

The involution $\sigma: X \rightarrow X$ is given by $\sigma(x, y)=(y, x)$. Then $\sigma$ is clearly a symplectic involution and its fixed point set is the diagram $\triangle(X)=S^{2}$. Then the quotient $X / \sigma=\mathbb{C} P^{2}$ is symplectic.

3. Seiberg-Witten invariant of the quotient manifold under an anti-symplectic involution with a 2-dimensional fixed point set. Let $X$ be a closed symplectic 4manifold with a symplectic structure $\omega$. A smooth map $\sigma: X \rightarrow X$ is an anti-symplectic involution if and only if it satisfies $\sigma^{*} \omega=-\omega$ and $\sigma^{2}=\operatorname{Id}$ on $X$. If $X$ is a Kähler surface then $\sigma$ is anti-symplectic if and only if $\sigma$ is anti-holomorphic; that is, $\sigma_{*} \circ J=-J \circ \sigma_{*}$ for the complex structure $J$ on $X$. For an example of an anti-holomorphic involution, we can consider a complex conjugation over a complex algebraic surface.

From now on suppose that there is an anti-symplectic involution $\sigma: X \rightarrow X$ with a 2-dimensional, compact submanifold $X^{\sigma}$ as a fixed point set. Then we have the following result.

LemmA 3.1. Each connected, oriented 2-dimensional component $\Sigma \subset X^{\sigma}$ is a Lagrangian surface.

Proof. Since $\sigma$ is anti-symplectic, $\sigma^{*} \omega=-\omega$ and so $\left.\sigma^{*} \omega\right|_{\Sigma}=-\left.\omega\right|_{\Sigma}$. However, over the fixed point set $\Sigma$, we have

$$
\left.\sigma^{*} \omega\right|_{\Sigma}=\left.\omega\right|_{\sigma(\Sigma)}=\left.\omega\right|_{\Sigma}
$$

Thus $\left.\omega\right|_{\Sigma}=0$ and $\Sigma$ is a Lagrangian surface in $X$. 
Proposition 3.2. For an anti-symplectic involution $\sigma$, we have $\sigma_{*} \circ J=-J \circ \sigma_{*}$ for a $\omega$-compatible almost complex structure $J$ as long as $\sigma$ is an isometry for the $\omega$-compatible metric $g$.

Proof. Let $g$ be a $\omega$-compatible metric such that $g(v, w)=\omega(v, J w)$, for all $v, w \in T X$, and $\omega$ is self-dual with respect to $g$.

Since $\sigma$ is anti-symplectic and acts as an isometry for the $\omega$-compatible metric $g$, we have

$$
\begin{aligned}
& g\left(J \sigma_{*} v, w\right)=\omega\left(\sigma_{*} v, w\right)=-\sigma^{*} \omega\left(\sigma_{*} v, w\right)=\omega\left(-v, \sigma_{*} w\right)=g\left(-J v, \sigma_{*} w\right) \\
& =\sigma^{*} g\left(-J v, \sigma_{*} w\right)=g\left(-\sigma_{*} J v, w\right), \quad \text { for all } v, w \in T X
\end{aligned}
$$

Thus we have $J \circ \sigma_{*}=-\sigma_{*} \circ J$ on $T X$.

LEMMA 3.3. Each connected, oriented 2-dimensional component $\Sigma \in X^{\sigma}$ satisfies $\chi(\Sigma)+\Sigma \cdot \Sigma=0$.

Proof. Let $J$ be the $\omega$-compatible almost complex structure and $g$ be the compatible metric.

Over $\left.T X\right|_{\Sigma}=T \Sigma \oplus N_{\Sigma}$, the induced map $\sigma_{*}$ acts as $\left.\sigma_{*}\right|_{T \Sigma}=$ Id and $\left.\sigma_{*}\right|_{N_{\Sigma}}=-$ Id where $T \Sigma$ and $N_{\Sigma}$ are the tangent and normal complex line bundle of $\Sigma$ in $X$, respectively. Then $\sigma$ acts as an isometry on $\left.T X\right|_{\Sigma}$ for the $\omega$-compatible metric $g$. Indeed, for all $v_{1}, v_{2} \in T \Sigma$ and $w_{1}, w_{2} \in N_{\Sigma}, g\left(v_{i}, w_{j}\right)=0$, for $i, j=1,2$, and

$$
\begin{aligned}
\sigma^{*} g\left(v_{1}, v_{2}\right) & =g\left(\sigma_{*} v_{1}, \sigma_{*} v_{2}\right)=g\left(v_{1}, v_{2}\right), \\
\sigma^{*} g\left(w_{1}, w_{2}\right) & =g\left(\sigma_{*} w_{1}, \sigma_{*} w_{2}\right)=g\left(-w_{1},-w_{2}\right)=g\left(w_{1}, w_{2}\right) .
\end{aligned}
$$

By Proposition 3.2, we have $J \circ \sigma_{*}=-\sigma_{*} \circ J$ on $\left.T X\right|_{\Sigma}$ and so $J$ is an orientation reversing isomorphism $J:\left.T_{x} \Sigma \rightarrow N_{\Sigma}\right|_{x}$, for each $x \in \Sigma$. Thus we have $\chi(\Sigma)=$ $-\Sigma \cdot \Sigma$.

THEOREM 3.4. Let $(X, \omega)$ be a symplectic 4-manifold and $\sigma: X \rightarrow X$ be an antisymplectic involution with a 2-dimensional compact submanifold as a fixed point set. If the fixed point set contains a Riemann surface $\Sigma$ with genus $g(\Sigma) \geq 2$ and $0 \neq[\Sigma] \in$ $H_{2}(X: \mathbb{Z})$, then the quotient manifold $X / \sigma$ with $b_{2}^{+}(X / \sigma)>1$ has a vanishing SeibergWitten invariant.

Proof. Let $\pi: X \rightarrow X / \sigma$ be the projection map and $\pi(\Sigma)=\Sigma^{\prime}$. By [5] and [24], we have $\Sigma^{\prime} \cdot \Sigma^{\prime}=2 \Sigma \cdot \Sigma$.

If the quotient $X / \sigma$ has a Seiberg-Witten basic class $L$ then, by Theorem 1.1, we have

$$
\left|c_{1}(L)\left[\Sigma^{\prime}\right]\right|+\Sigma^{\prime} \cdot \Sigma^{\prime} \leq-\chi\left(\Sigma^{\prime}\right) .
$$

By Lemma 3.3, $\chi(\Sigma)+\Sigma \cdot \Sigma=0$ and so the equation (1) implies that

$$
\left|c_{1}(L)\left[\Sigma^{\prime}\right]\right|+2 \Sigma \cdot \Sigma+\chi(\Sigma)=\left|c_{1}(L)\left[\Sigma^{\prime}\right]\right|+\Sigma \cdot \Sigma \leq 0 .
$$

Then we have

$$
\left|c_{1}(L)\left[\Sigma^{\prime}\right]\right| \leq-\Sigma \cdot \Sigma .
$$


Since $g(\Sigma) \geq 2$, we have $\Sigma \cdot \Sigma=-\chi(\Sigma)>0$ and so equation (2) yields a contradiction. Thus there is no Seiberg-Witten basic class over $X / \sigma$.

REMARK 3.5. Let $X$ be a closed, smooth, almost complex 4-manifold with an almost complex structure $J$. Assume that $\sigma: X \rightarrow X$ is an anti-holomorphic involution with a 2-dimensional, compact submanifold as a fixed point set. Then, since $J \circ \sigma_{*}=-\sigma_{*} \circ J$ on $T X$, we have an orientation reversing isomorphism $J$ : $\left.T_{x} \Sigma \rightarrow N_{\Sigma}\right|_{x}$, for all $x \in \Sigma$.

With the same conditions on $\Sigma$ as in Theorem 3.4, the quotient $X / \sigma$ with $b_{2}^{+}(X / \sigma)>1$ has a vanishing Seiberg-Witten invariant.

4. Relationship between Seiberg-Witten invariants on $X$ and $X^{\prime}$ when $g(\Sigma)=1$ and $\Sigma \cdot \Sigma=0$. Let $X^{\prime}$ be a closed smooth 4-manifold and $\pi: X \rightarrow X^{\prime}$ be a double branched cover along a surface $\Sigma^{\prime}$. Ruan and Wang [21] established a formula between the Seiberg-Witten invariants on $X$ and $X^{\prime}$ with $b_{2}^{+}\left(X^{\prime}\right), b_{2}^{+}(X)>1$ when $\Sigma^{\prime}$ has genus greater than 1 and $\Sigma^{\prime} \cdot \Sigma^{\prime}=0$. Suppose that $H_{2}(X ; \mathbb{Z})$ has no 2-torsion. Let $\pi^{-1}\left(\Sigma^{\prime}\right)=\Sigma$ and $Y_{0}$ be the complement of a tubular neighborhood of $\Sigma^{\prime}$.

THEOREM 4.1. [21]. Let $\pi: X \rightarrow X^{\prime}$ be a double cover branched along a surface $\Sigma^{\prime}$ with genus greater than $1,\left[\Sigma^{\prime}\right]^{2}=0$, and such that $b_{2}^{+}\left(X^{\prime}\right), b_{2}^{+}(X)>1$. Suppose that $\xi$ is a Spin ${ }^{c}$-structure on $X^{\prime}$ satisfying $c_{1}(\operatorname{det} \xi) \cdot\left[\Sigma^{\prime}\right] \leq 0$, and the virtual dimension of the Seiberg-Witten moduli space and the adjunction term, $\left|c_{1}(\operatorname{det} \xi)\left[\Sigma^{\prime}\right]\right|+\Sigma^{\prime} \cdot \Sigma^{\prime}+\chi\left(\Sigma^{\prime}\right)$ both vanish. Moreover let $\tilde{\xi}$ be a Spinc-structure on $X$ whose determinant bundle is $\operatorname{det} \tilde{\xi}=\pi^{*}(\operatorname{det} \xi) \otimes P D[\Sigma]^{-1}$ and whose restriction to $\tilde{Y}_{0}=\pi^{-1}\left(Y_{0}\right)$ is the pull-back of $\left.\xi\right|_{Y_{0}}$. Then the following equality holds:

$$
S W_{X}(\tilde{\xi})=S W_{X^{\prime}}(\xi)+k_{\xi}\left(X^{\prime}, \Sigma^{\prime}\right) \bmod 2,
$$

where $k_{\xi}\left(X^{\prime}, \Sigma^{\prime}\right)=\Sigma_{[\gamma] \in K_{Y}^{*}} \mathcal{S} W_{c}\left(\left.\xi\right|_{Y} \otimes \gamma\right)$ is an invariant of the triple $\left(X^{\prime}, \Sigma^{\prime}, \frac{\left[\Sigma^{\prime}\right]}{2}\right)$ and $c=c_{1}(\operatorname{det} \xi)^{2}-2 \chi\left(\Sigma^{\prime}\right)$.

Ruan and Wang proved Theorem 4.1 by using the relative Seiberg-Witten invariants formula [19]. Their idea is to rewrite the Seiberg-Witten invariants on $X$ and $X^{\prime}$ in terms of relative Seiberg-Witten invariants and relate the relative Seiberg-Witten invariants using the Seiberg-Witten theory with a $\mathbb{Z}_{2}$-action. Under the conditions of Theorem 4.1, all finite energy solutions of the Seiberg-Witten moduli space defined over the cylindrical end space are irreducible. They exclude the case in which $\Sigma^{\prime}$ is a torus, because here we have reducible solutions of the Seiberg-Witten equations over the cylindrical extensions of the complement of $T^{2}$.

In Section 4, we prove a formula between the Seiberg-Witten invariants of $X$ and $X^{\prime}$ when the genus of $\Sigma^{\prime}$ is 1 and $\Sigma^{\prime} \cdot \Sigma^{\prime}=0$ by using [18] and [21].

Assume that $X^{\prime}$ is a closed, smooth, oriented 4-manifold with a smoothly embedded 2-torus $T^{2}$ with self-intersection number 0 . Then $X^{\prime}$ is diffeomorphic to a 4-manifold $Y \cup_{\phi}\left(D^{2} \times T^{2}\right)$, where $Y$ is a smooth, compact, oriented 4-manifold with boundary $\partial Y \cong T^{3}, \phi: \partial\left(D^{2} \times T^{2}\right) \rightarrow \partial Y$ is an orientation reversing diffeomorphism and $D^{2}$ is a disk in $\mathbb{R}^{2}$ with $\partial D^{2} \cong S^{1}$. We identify $X^{\prime}=Y \cup_{\phi}\left(D^{2} \times T^{2}\right)$.

By Hirzebruch [14], if $\left[T^{2}\right]=2 a, a \in H_{2}\left(X^{\prime} ; \mathbb{Z}\right)$, then there is a branched double cover $\pi: X \rightarrow X^{\prime}$ along $\left[T^{2}\right]$. Then $X$ is a closed, oriented, smooth 4-manifold with a smoothly embedded torus $\tilde{T}^{2}=\pi^{-1}\left(T^{2}\right)$ with self-intersection number 0 and $X$ is 
diffeomorphic to $\tilde{Y} \cup_{\psi}\left(D^{2} \times \tilde{T}^{2}\right)$, where $\tilde{Y}$ is an unramified 2-fold cover of $Y$ with $\partial \tilde{Y}=\pi^{-1}(\partial Y)$ and $\psi: \partial\left(D^{2} \times \tilde{T}^{2}\right) \rightarrow \partial(\tilde{Y})$ is an orientation reversing diffeomorphism.

Suppose that $b_{2}^{+}(Y), b_{2}^{+}(\tilde{Y})>1$. Since $b_{2}^{+}\left(X^{\prime}\right)=b_{2}^{+}(Y)+1$ and $b_{2}^{+}(X)=b_{2}^{+}(\tilde{Y})+1$, we have $b_{2}^{+}\left(X^{\prime}\right), b_{2}^{+}(X)>2$.

Let $\tilde{\gamma}$ be a representative of the homology class $\psi_{*}\left[\partial D^{2} \times\{\mathrm{pt}\}\right] \in H_{1}(\partial \tilde{Y} ; \mathbb{Z})$. Suppose that $\tilde{\gamma} \in \operatorname{ker}\left(\tilde{i}_{*}\right)$, where $\tilde{i}_{*}: H_{1}(\partial \tilde{Y} ; \mathbb{R}) \rightarrow H_{1}(\tilde{Y} ; \mathbb{R})$ is induced from the inclusion $\tilde{i}: \partial \tilde{Y} \rightarrow \tilde{Y}$.

Then we can fix a $\tilde{b} \in H_{2}(\tilde{Y}, \partial \tilde{Y} ; \mathbb{R})$ such that the boundary $\partial \tilde{b}=\tilde{\gamma}$ and $\pi_{*} \tilde{\gamma}=\gamma=\phi_{*}\left(2\left[\partial D^{2} \times\{\mathrm{pt}\}\right]\right) \in \operatorname{ker}\left(i_{*}\right)$. Thus there exists $\pi_{*} \tilde{b}=b \in H_{2}(Y, \partial Y ; \mathbb{R})$ such that $\partial b=\gamma$, where $i_{*}: H_{1}(\partial Y ; \mathbb{R}) \rightarrow H_{1}(Y ; \mathbb{R})$.

Let $\xi$ be a Spin ${ }^{c}$-structure over $X^{\prime}$ with determinant bundle det $\xi=L$ and the restriction $\left.\xi\right|_{D^{2} \times T^{2}}$ of $\xi$ to $D^{2} \times T^{2}$ is trivial. Take $Y_{0}=X^{\prime} \backslash\left(D^{2} \times T^{2}\right)$ and $\tilde{Y}_{0}=\pi^{-1}\left(Y_{0}\right)$.

LEMMA 4.2. In the same situations as above, there exists a Spin ${ }^{c}$-structure $\tilde{\xi}$ on $X$ whose restriction to $D^{2} \times \tilde{T}^{2}$ is trivial, $\operatorname{det} \tilde{\xi}=\tilde{L} \cong \pi^{*} L \otimes P D^{-1}\left[\tilde{T}^{2}\right]$ and $\left.\tilde{\xi}\right|_{\tilde{Y}_{0}} \cong \pi^{*}\left(\left.\xi\right|_{Y_{0}}\right)$.

Proof. For the existence of the $\operatorname{Spin}^{c}$-structure $\xi$ with determinant bundle $\pi^{*} L \otimes \mathrm{PD}^{-1}\left[\tilde{T}^{2}\right]$ and $\left.\left.\pi^{*} \xi\right|_{Y_{0}} \cong \tilde{\xi}\right|_{\tilde{Y}_{0}}$, see Proposition 5.11 [21]. We only check that $\left.\tilde{\xi}\right|_{D^{2} \times \tilde{T}^{2}}$ is trivial.

The subspace $D^{2} \times \tilde{T}^{2}$ is a symplectic 4-manifold with a symplectic structure $\omega=d x_{1} d x_{2}+d y_{1} d y_{2}$, where $\left(x_{1}, x_{2}\right) \in \tilde{T}^{2}$ and $\left(y_{1}, y_{2}\right) \in D^{2}$ are coordinates. Then the positive spinor field $W^{+}(\tilde{\xi})$ and the determinant bundle $\tilde{L}$ over $D^{2} \times \tilde{T}^{2}$ can be decomposed by

$$
\left.W^{+}(\tilde{\xi})\right|_{D^{2} \times \tilde{T}^{2}}=E \otimes\left(\mathrm{II} \oplus K_{D^{2} \times \tilde{T}^{2}}^{*}\right),\left.\quad \tilde{L}\right|_{D^{2} \times \tilde{T}^{2}}=E^{2} \otimes K_{D^{2} \times \tilde{T}^{2}}^{*},
$$

for some complex line bundle $E \rightarrow D^{2} \times \tilde{T}^{2}$, where $K_{D^{2} \times \tilde{T}^{2}}$ is the canonical class and II is a trivial line bundle over $D^{2} \times \tilde{T}^{2}$.

Since $\left.L\right|_{D^{2} \times T^{2}}$ is trivial and $\left.\tilde{L}\right|_{D^{2} \times \tilde{T}^{2}}=\left.\left.\pi^{*} L\right|_{D^{2} \times \tilde{T}^{2}} \otimes \operatorname{PD}^{-1}\left[\tilde{T}^{2}\right]\right|_{D^{2} \times \tilde{T}^{2}},\left.\tilde{L}\right|_{D^{2} \times \tilde{T}^{2}}$ is trivial and $2 c_{1}(E)=c_{1}\left(K_{D^{2} \times \tilde{T}^{2}}\right)$.

Because

$$
c_{1}\left(K_{D^{2} \times \tilde{T}^{2}}\right)\left[D^{2} \times \tilde{T}^{2}\right]=-c_{1}\left(T\left(D^{2} \times \tilde{T}^{2}\right)\right)\left[D^{2} \times \tilde{T}^{2}\right]=-\left(\chi\left(\tilde{T}^{2}\right)+\tilde{T}^{2} \cdot \tilde{T}^{2}\right)=0,
$$

$\left.W^{+}(\tilde{\xi})\right|_{D^{2} \times \tilde{T}^{2}}$ and $\left.\tilde{L}\right|_{D^{2} \times \tilde{T}^{2}}$ are all trivial, where $T\left(D^{2} \times \tilde{T}^{2}\right)$ is the tangent bundle of $D^{2} \times \tilde{T}^{2}$. Since $W^{+}(\tilde{\xi})=\tilde{\xi} \times \operatorname{Spin}^{c}(4) \mathbb{C}^{2}$, we conclude that $\left.\tilde{\xi}\right|_{D^{2} \times \tilde{T}^{2}}$ is trivial.

From now on let $\left.\xi\right|_{Y_{0}}=\xi_{0}, \tilde{\xi}_{0}=\pi^{*}\left(\xi_{0}\right)$, $\operatorname{det}\left(\xi_{0}\right)=L_{0}$, and $\operatorname{det}\left(\tilde{\xi}_{0}\right)=\tilde{L}_{0}$. Denote $\tilde{Y}^{\prime}=\tilde{Y} \cup_{T^{3}} T^{3} \times[0, \infty)=\operatorname{cl}\left(\tilde{Y}_{0}\right) \cup_{T^{3}} T^{3} \times[0, \infty)$. Fix a flat metric $\tilde{h}$ on $T^{3}$ and a corresponding cylindrical end metric $\tilde{g}$ on $\tilde{Y}^{\prime}$ such that $\tilde{g}=\tilde{h}+d t^{2}$ near the end of $\tilde{Y}^{\prime}, t \in[0, \infty)$.

Since $\left.\tilde{\xi}_{0}\right|_{T^{3}}$ is trivial, $\tilde{\xi}_{0}$ is a $\operatorname{Spin}^{c}$-structure on $\tilde{Y}^{\prime}$. Over the space $\mathcal{A}\left(\tilde{L}_{0}\right) \times$ $\Gamma\left(W_{\tilde{Y}^{\prime}}^{+}\left(\tilde{\xi}_{0}\right)\right)$, we define Seiberg-Witten equations. For any compactly supported, realvalued, smooth, self-dual two-form $\tilde{\zeta} \in \Omega_{+}{ }^{2}\left(\tilde{Y}^{\prime} ; \mathbb{R}\right)$, let $\mathcal{M}_{\tilde{Y}^{\prime}}\left(\tilde{\xi}_{0}, \tilde{g}, \tilde{\zeta}\right)$ be the moduli space of all finite energy solutions of the perturbed Seiberg-Witten equations by the action of the gauge group, where the energy of a pair $(A, \psi)$ is defined by $\int_{\tilde{Y}^{\prime}}\left|F_{A}\right|^{2} \mathrm{dvol}$. Then, by [18], there is a continuous map $\tilde{\partial}_{\infty}$ and a covering map $\tilde{p}$

$$
\mathcal{M}_{\tilde{Y}^{\prime}}\left(\tilde{\xi}_{0}, \tilde{g}, \tilde{\zeta}\right) \stackrel{\tilde{\partial}_{\infty}}{\longrightarrow} \chi_{0}\left(T^{3}\right) \stackrel{\tilde{p}}{\longrightarrow} \chi\left(T^{3}\right)
$$


where $\chi\left(T^{3}\right)$ is the moduli space of the 3-dimensional Seiberg-Witten equations for the trivial $\mathrm{Spin}^{c}$-structure and a flat metric over $T^{3}$ and $\chi_{0}\left(T^{3}\right)$ is a covering space of $\chi\left(T^{3}\right)$ defined in Section 2 [18].

By Lemma $2.3[\mathbf{1 8}]$, there is a unique singular point $\tilde{\theta}=\left(\tilde{\theta}_{0}, 0\right) \in \chi\left(T^{3}\right)$ such that $\operatorname{ker} D_{\tilde{\theta}_{0}} \neq 0$ and $\mathcal{M}_{\tilde{Y}}\left(\tilde{\mathcal{L}}_{0}, \tilde{g}, \tilde{\zeta}\right)$ has singularities induced from $\tilde{\theta}$ and it is a compact manifold with boundary, the boundary mapping to the singular point $\tilde{\theta}$.

Let $\mathcal{C}_{\tilde{Y}}$ be the set of isomorphism classes of $\operatorname{Spin}^{c}$-structures $\tilde{\xi}_{0}$ on the space $\tilde{Y}=\operatorname{cl}\left(X \backslash\left(D^{2} \times \tilde{T}^{2}\right)\right)$ such that $\left.\tilde{\xi}_{0}\right|_{\partial \tilde{Y}}$ is trivial. By [18], $\tilde{p}^{-1}(\tilde{\theta})$ is in one-to-one correspondence with the set $\tilde{r}^{-1}\left(\tilde{\xi}_{0}\right)$, where $\tilde{r}: \mathcal{C}_{\tilde{Y}, \partial \tilde{Y}} \rightarrow \mathcal{C}_{\tilde{Y}}$ is the forgetful map. For the $\operatorname{set} \mathcal{C}_{\tilde{Y}, \partial \tilde{Y}}$, see [18].

By definition [18], the $\operatorname{Spin}^{c}$-structure $\tilde{\xi}^{\prime} \in \wedge^{+}(\tilde{\xi})$ if and only if $\tilde{\xi}^{\prime} \in \tilde{r}^{-1}\left(\tilde{\xi}_{0}\right)$ and for all points in $\tilde{T}^{2}$,

$$
\left\langle c_{1}(\tilde{L}), \tilde{b}+\left[D^{2} \times \mathrm{pt}\right]\right\rangle<\left\langle c_{1}\left(\operatorname{det} \tilde{\xi}^{\prime}\right), \tilde{b}\right\rangle .
$$

Then the relative Seiberg-Witten invariant $\mathcal{S} W_{\tilde{Y}^{\prime}}\left(\tilde{\xi}_{0}\right)$ over $\tilde{Y}^{\prime}$ is defined by

$$
\mathcal{S} W_{\tilde{Y}^{\prime}}\left(\tilde{\xi}_{0}\right)=\Sigma_{\tilde{\xi}^{\prime} \in \wedge^{+}(\tilde{\xi})} \sharp\left(\mathcal{M}_{\tilde{Y}^{\prime}}\left(\tilde{\xi}_{0}, \tilde{g}, \tilde{\zeta}\right) \cap \tilde{D} \cap \tilde{\partial}_{\infty}^{-1}\left(\tilde{\theta}_{\tilde{\xi}^{\prime}}\right)\right) ;
$$

that is the sum of the counting numbers of a smooth, compact, zero-dimensional manifold $\mathcal{M}_{\tilde{Y}^{\prime}}\left(\tilde{\xi}_{0}, \tilde{g}, \tilde{\zeta}\right) \cap \tilde{D} \cap \tilde{\partial}_{\infty}^{-1}\left(\tilde{\theta}_{\tilde{\xi}^{\prime}}\right)$, where $\tilde{\theta}_{\tilde{\xi}^{\prime}} \in p^{-1}(\tilde{\theta})$ is the element corresponding to $\tilde{\xi}^{\prime} \in \tilde{r}^{-1}\left(\tilde{\xi}_{0}\right)$.

REMARK 4.3. $\tilde{D}$ is a geometric representative of $\tilde{\mu}(\mathrm{pt})^{\frac{\tilde{d}}{2}}$ that is similar to the geometric representative defined in the Donaldson invariant and $\tilde{\mu}$ is a map

$$
\tilde{\mu}: H_{0}\left(\tilde{Y}^{\prime} ; \mathbb{Q}\right) \rightarrow H^{2}\left(\mathcal{M}_{\tilde{Y}^{\prime}}\left(\tilde{\xi}_{0}, \tilde{g}, \tilde{\zeta}\right) ; \mathbb{Q}\right)
$$

defined by $\tilde{\mu}(\mathrm{pt})=c_{1}(\mathbb{L})$, where $\mathbb{L}=\pi^{*}\left(\tilde{L}_{0}\right)$ is the bundle over $\mathcal{M}_{\tilde{Y}^{\prime}}\left(\tilde{\xi}_{0}, \tilde{g}, \tilde{\zeta}\right) \times \tilde{Y}^{\prime}$, $\pi: \mathcal{M}_{\tilde{Y}}\left(\tilde{\xi}_{0}, \tilde{g}, \tilde{\zeta}\right) \times \tilde{Y}^{\prime} \rightarrow \tilde{Y}^{\prime}$ is the projection map and $\tilde{d}$ is given by $\tilde{d}=\frac{1}{4}\left(c_{1}\left(\tilde{L}_{0}\right)^{2}-2 \chi(\tilde{Y})-3 \operatorname{Sign}(\tilde{Y})\right)$.

Since $X$ is a 2 -fold branched cover of $X^{\prime}$, there is an involution $\sigma: X \rightarrow X$ with a fixed point set $\tilde{T}^{2}$. Then $\sigma$ acts freely over $\tilde{Y}^{\prime}$ and there are involutions $\tilde{\tau}: \tilde{\xi}_{0}=\pi^{*} \xi_{0} \rightarrow \tilde{\xi}_{0}$ and $\tau=\operatorname{det} \tilde{\tau}: \tilde{L}_{0} \rightarrow \tilde{L}_{0}$ induced from the involution $\sigma_{*}$ on the orthonormal frame bundle of $\tilde{Y}^{\prime}$. Then we have an involution

$$
\tau^{*}: \mathcal{M}_{\tilde{Y}^{\prime}}\left(\tilde{\xi}_{0}, \tilde{g}, \tilde{\zeta}\right) \rightarrow \mathcal{M}_{\tilde{Y}^{\prime}}\left(\tilde{\xi}_{0}, \tilde{g}, \tilde{\zeta}\right)
$$

and a $\mathbb{Z}_{2}$-invariant moduli space $\mathcal{M}_{\tilde{Y}}\left(\tilde{\xi}_{0}, \tilde{g}, \tilde{\zeta}\right)^{\mathbb{Z}_{2}}$, which is the fixed point set of $\tau^{*}$ and is independent of the choice of $\tau$.

Proposition 4.4. The maps $\tilde{\partial}_{\infty}$ and $\tilde{p}$ are $\mathbb{Z}_{2}$-equivariant. Furthermore the singular point $\tilde{\theta} \in \chi\left(T^{3}\right)^{\mathbb{Z}_{2}}$.

Proof. Every $\operatorname{Spin}^{c}$-structure $\xi_{\mathbb{R} \times T^{3}} \rightarrow \mathbb{R} \times T^{3}$ is a pull back from a $\operatorname{Spin}^{c}$ structure $\xi_{T^{3}}$ on $T^{3}$. From the embedding $\operatorname{Spin}^{c}(3) \rightarrow \operatorname{Spin}^{c}(4)$ sending $(q, x)$ to $(q, q, x)$, we have an identification between the positive and negative spinor spaces $W^{+}\left(\xi_{\mathbb{R} \times T^{3}}\right) \cong W^{-}\left(\xi_{\mathbb{R} \times T^{3}}\right) \cong \pi^{*} \xi_{T^{3}}$.

Let det $\xi_{\mathbb{R} \times T^{3}}=L_{\mathbb{R} \times T^{3}}$ and $\operatorname{det} \xi_{T^{3}}=L_{T^{3}}$. Then there is a gauge transformation $g$ : $\mathbb{R} \times T^{3} \rightarrow S^{1}$ such that for all connections $A \in \mathcal{A}\left(L_{\mathbb{R} \times T^{3}}\right), g(A)$ has no $d t$-component, $t \in \mathbb{R}$, which is said to be in temporal gange. Then the Seiberg-Witten equations over 
$\mathbb{R} \times T^{3}$ can be written as the gradient flow equations. The critical points of the gradient flow equation are solutions of 3-dimensional Seiberg-Witten equations over $T^{3}$.

Thus if we consider a $\mathbb{Z}_{2}$-invariant solution of the Seiberg-Witten equations over $\mathbb{R} \times T^{3}$, then it induces a $\mathbb{Z}_{2}$-invariant solution of the 3 -dimensional Seiberg-Witten equations over $T^{3}$. Thus we have the restrictions of $\tilde{\partial}_{\infty}$ and $\tilde{\pi}$ such that

$$
\mathcal{M}_{\tilde{Y}^{\prime}}\left(\tilde{\xi}_{0}, \tilde{g}, \tilde{\zeta}\right)^{\mathbb{Z}_{2}} \stackrel{\tilde{\partial}_{\infty}}{\longrightarrow} \chi_{0}\left(T^{3}\right)^{\mathbb{Z}_{2}} \stackrel{\tilde{p}}{\longrightarrow} \chi\left(T^{3}\right)^{\mathbb{Z}_{2}}
$$

If there is a $u \neq 0$ in $\operatorname{ker} D_{\tilde{\theta}_{0}}$, then $h(u) \neq 0$, for all $h \in \mathbb{Z}_{2}$, because $h$ is an involution. Since the Dirac operator $D_{A}$ is $\mathbb{Z}_{2}$-equivariant, $D_{h\left(\tilde{\theta}_{0}\right)} h(u)=h\left(D_{\tilde{\theta}_{0}} u\right)=0$, for all $h \in \mathbb{Z}_{2}$. Thus there is a $h(u) \neq 0$ such that $h(u) \in \operatorname{ker} D_{h\left(\tilde{\theta}_{0}\right)}$. Since $\tilde{\theta}=\left(\tilde{\theta}_{0}, 0\right)$ is the unique point such that $\operatorname{ker} D_{\tilde{\theta}_{0}} \neq 0$, we conclude that, for all $h \in \mathbb{Z}_{2}, h\left(\tilde{\theta}_{0}\right)=\tilde{\theta}_{0}$ in $\chi\left(T^{3}\right)$.

REMARK 4.5. By [5] and [21], we can choose a generic, $\mathbb{Z}_{2}$-invariant Riemannian metric $\tilde{g}$ and a $\mathbb{Z}_{2}$-invariant self-dual two-form $\tilde{\zeta}$ over $\tilde{Y}^{\prime}$ such that $\left(\mathcal{M}_{\tilde{Y}^{\prime}}\left(\tilde{\xi}_{0}, \tilde{g}, \tilde{\zeta}\right)^{\mathbb{Z}_{2}} \cap D \cap\left(\tilde{\partial}_{\infty}\right)^{-1}\left(\tilde{\theta}_{\tilde{\xi}^{\prime}}\right)\right)$ is a smooth, compact, zero-dimensional manifold, where $D$ is the geometric representative of $\mu(\mathrm{pt})^{\frac{\tilde{d}}{4}}, \mu$ is given by $\mu: H_{0}\left(\tilde{Y}^{\prime} ; \mathbb{Q}\right) \rightarrow H^{2}\left(\mathcal{M}_{\tilde{Y}^{\prime}}\left(\tilde{\xi}_{0}, \tilde{g}, \tilde{\zeta}\right)^{\mathbb{Z}_{2}} ; \mathbb{Q}\right)$ and $\operatorname{dim} \mathcal{M}_{\tilde{Y}^{\prime}}(\tilde{\xi} 0, \tilde{g}, \tilde{\zeta})^{\mathbb{Z}_{2}}=\frac{\tilde{d}}{2}$ smooth.

However, in this case, the space $\left(\mathcal{M}_{\tilde{Y}^{\prime}}\left(\tilde{\xi}_{0}, \tilde{g}, \tilde{\zeta}\right) \cap \tilde{D} \cap \tilde{\partial}_{\infty}^{-1}\left(\tilde{\theta}_{\tilde{\xi}^{\prime}}\right)\right)$ may not be

As in Theorem 3.8 of [21], by comparing the $\mathbb{Z}_{2}$-invariant moduli space over $\tilde{Y}^{\prime}$ with the moduli space over $Y^{\prime}$, for $\tilde{g}=p^{*} g$ and $\tilde{\zeta}=p^{*} \zeta$, there is a homeomorphism

$$
\mathcal{M}_{\tilde{Y}^{\prime}}\left(\tilde{\xi}_{0}, \tilde{g}, \tilde{\zeta}\right)^{\mathbb{Z}_{2}} \cong \mathcal{M}_{Y^{\prime}}\left(\xi_{0}, g, \zeta\right) \amalg\left(\amalg_{\eta \in \mathcal{K}_{Y^{\prime}}} \mathcal{M}_{Y^{\prime}}\left(\xi_{0} \otimes \eta, g, \zeta\right)\right),
$$

where $\mathcal{K}_{Y^{\prime}}$ is a subspace of $H^{2}\left(Y^{\prime} ; \mathbb{Z}\right)$ consisting of isomorphic line bundles $\eta$ on $Y^{\prime}$ that pull back to the trivial line bundle on $\tilde{Y}^{\prime}$ and $\left.\eta\right|_{T^{3}}$ is trivial.

Then by [18] there are continuous maps $\partial_{\infty}: \mathcal{M}_{Y^{\prime}}\left(\xi_{0}, g, \zeta\right) \rightarrow \chi_{0}\left(T^{3}\right)$ and $\partial_{\infty}^{\prime}: \mathcal{M}_{Y^{\prime}}\left(\xi_{0} \otimes \eta, g, \zeta\right) \rightarrow \chi_{0}\left(T^{3}\right)$

Let $D^{\prime}$ and $D^{\prime \prime}$ be geometric representatives of $\mu(\mathrm{pt}) \in H^{2}\left(\mathcal{M}_{Y^{\prime}}\left(\xi_{0}, g, \zeta\right): \mathbb{Q}\right)$ and $\mu^{\prime}(\mathrm{pt}) \in H^{2}\left(\mathcal{M}_{Y^{\prime}}\left(\xi_{0} \otimes \eta, g, \zeta\right): \mathbb{Q}\right)$, respectively.

Then we can define the relative Seiberg-Witten invariant on $Y^{\prime}$ by

$$
\begin{aligned}
\mathcal{S} W_{Y^{\prime}}\left(\xi_{0}\right) & =\Sigma_{\xi^{\prime} \in \wedge^{+}(\xi)} \sharp\left(\mathcal{M}_{Y^{\prime}}\left(\xi_{0}, g, \zeta\right) \cap D^{\prime} \cap \partial_{\infty}^{-1}\left(\theta_{\xi^{\prime}}\right)\right), \\
\mathcal{S} W_{Y^{\prime}}\left(\xi_{0} \otimes \eta\right) & =\Sigma_{\xi_{\eta} \wedge^{\prime} \in \wedge^{+}(\xi \otimes \eta \tilde{~})} \sharp\left(\mathcal{M}_{Y^{\prime}}\left(\xi_{0} \otimes \eta, g, \zeta\right) \cap D^{\prime \prime} \cap \partial_{\infty}^{\prime}{ }^{-1}\left(\theta_{\xi_{\eta}}\right)\right),
\end{aligned}
$$

where $\tilde{\eta} \rightarrow X$ is an extension of the bundle $\eta \rightarrow Y^{\prime}$.

By Proposition 4.4 , we can define the $\mathbb{Z}_{2}$-invariant, relative Seiberg-Witten invariant $\mathcal{S} W_{\tilde{Y}^{\prime}}\left(\tilde{\xi}_{0}\right)^{\mathbb{Z}_{2}}$ by

$$
\mathcal{S} W_{\tilde{Y}^{\prime}}\left(\tilde{\xi}_{0}\right)^{\mathbb{Z}_{2}}=\Sigma_{\tilde{\xi}^{\prime} \in \wedge^{+}(\tilde{\xi})} \sharp\left(\mathcal{M}_{\tilde{Y}^{\prime}}\left(\tilde{\xi}_{0}, \tilde{g}, \tilde{\zeta}\right)^{\mathbb{Z}_{2}} \cap D \cap\left(\tilde{\partial}_{\infty}\right)^{-1}\left(\tilde{\theta}_{\tilde{\xi}^{\prime}}\right)\right),
$$

where $\pi^{*} \xi^{\prime}=\pi^{*} \xi_{\eta}^{\prime}=\tilde{\xi}^{\prime}$.

By using the method of proof as in Theorem 2.2 of [21], for a generic, $\mathbb{Z}_{2}$-invariant, self-dual two-form $\tilde{\zeta}$, we have

$$
\mathcal{S} W_{\tilde{Y}^{\prime}}\left(\tilde{\xi}_{0}\right)^{\mathbb{Z}_{2}}=\mathcal{S} W_{\tilde{Y}^{\prime}}\left(\tilde{\xi}_{0}\right) \bmod 2 .
$$

Let $\operatorname{det} \tilde{\xi}^{\prime}=\tilde{L}^{\prime}, \operatorname{det} \xi^{\prime}=L^{\prime}$, and $\operatorname{det} \xi_{\eta}^{\prime}=L_{\eta}^{\prime}$. 
Proposition 4.6. $\tilde{\xi}^{\prime} \in \wedge^{+}(\tilde{\xi})$ if and only if

$$
\xi^{\prime} \in \wedge^{+}(\xi) \quad \text { and }\left\langle c_{1}\left(L_{\eta}^{\prime}\right), \pi_{*} \tilde{b}\right\rangle>\left\langle c_{1}(L), \pi_{*} \tilde{b}+\pi_{*}\left[D^{2} \times p t\right]\right\rangle .
$$

Proof. For all $A \in \mathcal{A}(\tilde{L})$ and all points pt $\in \tilde{T}^{2}$, we have

$$
\left\langle c_{1}(\tilde{L}), \tilde{b}+\left[D^{2} \times \mathrm{pt}\right]\right\rangle=\int_{\tilde{b}+\left[D^{2} \times \mathrm{pt}\right]} \frac{i}{2 \pi} F_{A} .
$$

Since $\left.\tilde{L}\right|_{D^{2} \times \tilde{T}^{2}}$ and $\left.L\right|_{D^{2} \times T^{2}}$ are trivial and $\pi^{*}\left(\xi_{0}\right) \cong \tilde{\xi}_{0}$, we have

$$
\begin{gathered}
\left\langle c_{1}(\tilde{L}), \tilde{b}+\left[D^{2} \times \mathrm{pt}\right]\right\rangle=\left\langle c_{1}\left(\tilde{L}_{0}\right), \tilde{b}\right\rangle, \\
\left\langle c_{1}\left(\tilde{L}_{0}\right), \tilde{b}\right\rangle=\left\langle\pi^{*} c_{1}\left(L_{0}\right), \tilde{b}\right\rangle=\left\langle c_{1}\left(L_{0}\right), \pi_{*} \tilde{b}\right\rangle=\left\langle c_{1}(L), \pi_{*} \tilde{b}+\pi_{*}\left[D^{2} \times \mathrm{pt}\right]\right\rangle .
\end{gathered}
$$

Thus we have

$$
\left\langle c_{1}(\tilde{L}), \tilde{b}+\left[D^{2} \times \mathrm{pt}\right]\right\rangle=\left\langle c_{1}(L), \pi_{*} \tilde{b}+\pi_{*}\left[D^{2} \times \mathrm{pt}\right]\right\rangle
$$

Furthermore,

$$
\begin{aligned}
& \left\langle c_{1}\left(\tilde{L}^{\prime}\right), \tilde{b}\right\rangle=\left\langle\pi^{*} c_{1}\left(L^{\prime}\right), \tilde{b}\right\rangle=\left\langle c_{1}\left(L^{\prime}\right), \pi_{*} \tilde{b}\right\rangle=\left\langle c_{1}\left(L^{\prime}\right), b\right\rangle, \\
& \left\langle c_{1}\left(\tilde{L}^{\prime}\right), \tilde{b}\right\rangle=\left\langle\pi^{*} c_{1}\left(L_{\eta}^{\prime}\right), \tilde{b}\right\rangle=\left\langle c_{1}\left(L_{\eta}^{\prime}\right), \pi_{*} \tilde{b}\right\rangle=\left\langle c_{1}\left(L_{\eta}^{\prime}\right), b\right\rangle,
\end{aligned}
$$

where $\partial b=\gamma \in$ keri $i_{*}$.

By equations (3) and (4) we conclude that $\tilde{\xi}^{\prime} \in \wedge^{+}(\xi)$ if and only if

$$
\xi^{\prime} \in \wedge^{+}(\xi) \text { and }\left\langle c_{1}\left(L_{\eta}^{\prime}\right), \pi_{*} \tilde{b}\right\rangle>\left\langle c_{1}(L), \pi_{*} \tilde{b}+\pi_{*}\left[D^{2} \times \mathrm{pt}\right]\right\rangle,
$$

where $\pi^{*} \xi^{\prime}=\pi^{*} \xi_{\eta}^{\prime}=\tilde{\xi}^{\prime}$.

Let $\mathbb{O}_{\eta}=\left\{\xi_{\eta}^{\prime} \in r^{-1}\left(\xi_{0} \otimes \eta\right) \mid\left\langle c_{1}\left(L_{\eta}^{\prime}, \pi_{*} \tilde{b}\right\rangle>\left\langle c_{1}(L), \pi_{*} \tilde{b}+\pi_{*}\left[D^{2} \times \mathrm{pt}\right]\right\rangle\right\}\right.$.

We now come to our main theorem.

THEOREM 4.7. Let $\pi: X \rightarrow X^{\prime}$ be a double cover branched along a torus $T^{2}$ with self-intersection 0 and $b_{2}^{+}\left(X^{\prime}\right), b_{2}^{+}(X)>2$. Suppose that $H_{2}(X ; \mathbb{Z})$ has no 2-torsion and $\xi$ is a Spinc ${ }^{-}$-structure on $X^{\prime}$ such that $\left.\xi\right|_{D^{2} \times T^{2}}$ is trivial. Let $\tilde{\xi}$ be a Spin ${ }^{c}$-structure on $X$ whose restriction to $D^{2} \times \tilde{T}^{2}$ is trivial, the determinant bundle $\tilde{L} \cong \pi^{*} L \otimes P D^{-1}\left[\tilde{T}^{2}\right]$ and $\left.\tilde{\xi}\right|_{\tilde{Y}_{0}} \cong \pi^{*}\left(\left.\xi\right|_{Y_{0}}\right)$. Then we have a relation between the Seiberg-Witten invariants of $X$ and those of $X^{\prime}$ such that

$$
\mathcal{S} W_{X}(\tilde{\xi})=\mathcal{S} W_{X^{\prime}}(\xi)+k\left(X^{\prime}, T^{2}, a\right) \bmod 2,
$$

where

$$
k\left(X^{\prime}, T^{2}, a\right)=\Sigma_{\eta \in \mathcal{K}_{Y^{\prime}}} \Sigma_{\xi_{\eta}^{\prime} \in \mathbb{O}_{\eta}} \sharp\left(\mathcal{M}_{Y^{\prime}}\left(\xi_{0} \otimes \eta, g, \zeta\right) \cap D^{\prime \prime} \cap\left(\partial_{\infty}^{\prime}\right)^{-1}\left(\theta_{\xi_{\eta}^{\prime}}\right)\right) .
$$

Proof. By Proposition 4.6 we have a homeomorphism between smooth, compact, zero-dimensional spaces

$$
\begin{aligned}
& \amalg_{\tilde{\xi}^{\prime} \in \wedge^{+}(\tilde{\xi})}\left(\mathcal{M}_{\tilde{Y}^{\prime}}\left(\tilde{\xi}_{0}, \tilde{g}, \tilde{\zeta}\right)^{\mathbb{Z}_{2}} \cap D \cap\left(\tilde{\partial}_{\infty}\right)^{-1}\left(\tilde{\theta}_{\tilde{\xi}^{\prime}}\right)\right) \\
& \cong \amalg_{\xi^{\prime} \in \wedge^{+}(\xi)}\left(\mathcal{M}_{Y^{\prime}}\left(\xi_{0}, g, \zeta\right) \cap D^{\prime} \cap\left(\partial_{\infty}\right)^{-1}\left(\theta_{\xi^{\prime}}\right)\right) \amalg, \\
& \amalg_{\eta \in \mathcal{K}_{Y^{\prime}}} \amalg_{\xi_{\eta}^{\prime} \in \mathbb{O}_{\eta}}\left(\mathcal{M}_{Y^{\prime}}\left(\xi_{0} \otimes \eta, g, \zeta\right) \cap D^{\prime \prime} \cap\left(\partial_{\infty}^{\prime}\right)^{-1}\left(\theta_{\xi_{\eta}^{\prime}}\right)\right),
\end{aligned}
$$

where $\pi^{*} \xi^{\prime}=\pi^{*} \xi_{\eta}^{\prime}=\tilde{\xi}^{\prime}$. 
Thus, under mod 2 we have

$$
\mathcal{S} W_{\tilde{Y}^{\prime}}\left(\tilde{\xi}_{0}\right)^{\mathbb{Z}_{2}}=\mathcal{S} W_{Y^{\prime}}\left(\xi_{0}\right)+\Sigma_{\eta \in \mathcal{K}_{Y^{\prime}}} \Sigma_{\xi_{\eta}^{\prime} \in \mathbb{O}_{\eta}} \sharp\left(\mathcal{M}_{Y^{\prime}}\left(\xi_{0} \otimes \eta, g, \zeta\right) \cap D^{\prime \prime} \cap\left(\partial_{\infty}^{\prime}\right)^{-1}\left(\theta_{\xi_{\eta}^{\prime}}\right)\right) .
$$

The equation (2) implies that

$$
\mathcal{S} W_{\tilde{Y}^{\prime}}\left(\tilde{\xi}_{0}\right)^{\mathbb{Z}_{2}}=\mathcal{S} W_{\tilde{Y}^{\prime}}\left(\tilde{\xi}_{0}\right) \bmod 2 .
$$

By using Theorem 4.1 of [18] we have

$$
\mathcal{S} W_{X}(\tilde{\xi})=\mathcal{S} W_{\tilde{Y}^{\prime}}\left(\tilde{\xi}_{0}\right), \quad \mathcal{S} W_{X^{\prime}}(\xi)=\mathcal{S} W_{Y^{\prime}}\left(\xi_{0}\right) \bmod 2 .
$$

From equations (5), (6), and (7) we conclude that under mod 2,

$$
\mathcal{S} W_{X}(\tilde{\xi})=\mathcal{S} W_{X^{\prime}}(\xi)+\Sigma_{\eta \in \mathcal{K}_{Y^{\prime}}} \Sigma_{\xi_{\eta}^{\prime} \in \mathbb{O}_{\eta}} \sharp\left(\mathcal{M}_{Y^{\prime}}\left(\xi_{0} \otimes \eta, g, \zeta\right) \cap D^{\prime \prime} \cap\left(\partial_{\infty}^{\prime}\right)^{-1}\left(\theta_{\xi_{\eta}^{\prime}}\right)\right),
$$

completing the proof.

REMARK 4.8. By definition, $\xi^{\prime \prime} \in \wedge^{+}(\xi \otimes \tilde{\eta})$ if and only if $\xi^{\prime \prime} \in r^{-1}\left(\left.(\xi \otimes \tilde{\eta})\right|_{Y}\right)=$ $r^{-1}\left(\xi_{0} \otimes \eta\right)$ and $\left\langle c_{1}\left(\operatorname{det} \xi^{\prime \prime}\right), \pi_{*} \tilde{b}\right\rangle>\left\langle c_{1}(\operatorname{det}(\xi \otimes \tilde{\eta})), \pi_{*} \tilde{b}+\pi_{*}\left[D^{2} \times \mathrm{pt}\right]\right\rangle$.

Because we do not know the action of the line bundle $\tilde{\eta}$ over $D^{2} \times T^{2}$ and $\xi_{\eta}^{\prime}$ in Theorem 4.7 only satisfies

$$
\left\langle c_{1}\left(L_{\eta}^{\prime}\right), \pi_{*} \tilde{b}\right\rangle>\left\langle c_{1}(L), \pi_{*} \tilde{b}+\pi_{*}\left[D^{2} \times \mathrm{pt}\right]\right\rangle,
$$

we conclude that in general, $\wedge^{+}(\xi \otimes \tilde{\eta}) \neq \mathbb{O}_{\eta}$ and

$$
\Sigma_{\xi_{\eta}^{\prime} \in \mathbb{O}_{\eta}} \sharp\left(\mathcal{M}_{Y^{\prime}}\left(\xi_{0} \otimes \eta, g, \zeta\right) \cap D^{\prime \prime} \cap\left(\partial_{\infty}^{\prime}\right)^{-1}\left(\theta\left(\xi_{\eta}^{\prime}\right)\right)\right) \neq \mathcal{S} W_{Y^{\prime}}\left(\xi_{0} \otimes \eta\right) .
$$

Thus $\Sigma_{\eta \in \mathcal{K}_{Y^{\prime}}} \Sigma_{\xi_{\eta}^{\prime} \in \mathbb{O}_{\eta}} \sharp\left(\mathcal{M}_{Y^{\prime}}\left(\xi_{0} \otimes \eta, g, \zeta\right) \cap D^{\prime} \cap\left(\partial_{\infty}^{\prime}\right)^{-1}\left(\theta_{\xi_{\eta}^{\prime}}\right)\right)$ cannot be extended to a Seiberg-Witten invariant $\mathcal{S} W_{X^{\prime}}(\xi \otimes \tilde{\eta})$ and, as in [21], it is an invariant of $\left(X^{\prime}, T^{2}, a\right)$, where $2 a=\left[T^{2}\right] \in 2 H_{2}\left(X^{\prime} ; \mathbb{Z}\right)$.

COROLlary 4.9. Let $X$ be a closed symplectic 4-manifold with $b_{2}^{+}(X)>2$. Suppose that $\sigma: X \rightarrow X$ is an anti-symplectic involution with a torus $T^{2}$ as a fixed point set. Under the conditions for the Spin ${ }^{c}$-structures $\xi$ and $\tilde{\xi}$ of Theorem 4.7 , we have a relation between the Seiberg-Witten invariants of $X$ and the quotient $X / \sigma=X^{\prime}$ with $b_{2}^{+}\left(X^{\prime}\right)>2$ such that

$$
\mathcal{S} W_{X}(\tilde{\xi})=\mathcal{S} W_{X^{\prime}}(\xi)+k\left(X^{\prime}, T^{2}, a\right) \bmod 2 .
$$

Proof. There is a branched double cover $\pi: X \rightarrow X^{\prime}$ along $\pi\left(T^{2}\right)=T^{2}$, and so the required result follows.

5. Applications. To find the relationship between the Seiberg-Witten invariants on $X$ and $X^{\prime}$ of Theorem 4.7, we have to calculate the invariant $k\left(X^{\prime}, T^{2}, a\right)$. As in [21] we can show that $k\left(X^{\prime}, T^{2}, a\right)=0$ for many cases.

Proposition 5.1. Let $X$ be a Kähler surface with $b_{2}^{+}(X)>3$ and with the canonical class $K_{X}$ satisfying $K_{X}^{2}>0$. Let $\sigma: X \rightarrow X$ be an anti-holomorphic involution with a smoothly embedded torus as a fixed point set. Then the Seiberg-Witten invariant on the 
quotient $X^{\prime}$ is

$$
\mathcal{S} W_{X}(\tilde{\xi})=\mathcal{S} W_{X^{\prime}}(\xi) \bmod 2
$$

for the Spin ${ }^{c}$-structures $\tilde{\xi}$ and $\xi$ of Theorem 4.7 .

Proof. Consider a projection map $\pi: X \rightarrow X^{\prime}=X / \sigma$. Then, by [24], we have $b_{2}^{+}(X)=2 b_{2}^{+}\left(X^{\prime}\right)+1$ and $b_{2}^{+}\left(X^{\prime}\right)>1$.

Since $\sigma$ acts freely on $Y^{\prime}$ and

$$
2 \chi\left(Y^{\prime}\right)+3 \operatorname{Sign}\left(Y^{\prime}\right)=2 \chi\left(X^{\prime}\right)+3 \operatorname{Sign}\left(X^{\prime}\right)=K_{X^{\prime}}^{2}>0,
$$

by [25] there is no reducible or irreducible solution of the Seiberg-Witten equations over the cylindrical end space $Y^{\prime}$. Thus the moduli space $\mathcal{M}_{Y^{\prime}}\left(\xi_{0} \otimes \eta, g, \mu\right)$ is empty and hence the invariant

$$
k\left(X^{\prime}, T^{2}, a\right)=\Sigma_{\xi_{\eta}^{\prime} \in \mathbb{O}} \Sigma_{\eta \in \mathcal{K}_{Y^{\prime}}} \sharp\left(\mathcal{M}_{Y^{\prime}}\left(\xi_{0} \otimes \eta, g, \mu\right) \cap D^{\prime} \cap \partial_{\infty}^{\prime-1}\left(\theta_{\xi_{\eta}^{\prime}}\right)\right)=0,
$$

for all cases. Thus the Seiberg-Witten invariant on the quotient $X^{\prime}$ is

$$
\mathcal{S} W_{X}(\tilde{\xi})=\mathcal{S} W_{X^{\prime}}(\xi) \bmod 2
$$

for the $\operatorname{Spin}^{c}$-structures $\tilde{\xi}$ and $\xi$ in Theorem 4.7.

In the case considered in [21], $g\left(\Sigma^{\prime}\right)>1$ and $\Sigma^{\prime} \cdot \Sigma^{\prime}=0$. They did not find an example such that $k\left(X^{\prime}, \Sigma^{\prime}, a\right) \neq 0 \bmod 2$ although they believe such an example should exist. When $g\left(\Sigma^{\prime}\right)=1$ and $\Sigma^{\prime} \cdot \Sigma^{\prime}=0$, there is an example such that $k\left(X^{\prime}, T^{2}, a\right) \neq 0$ $\bmod 2$.

EXAMPLE 5.2. Let $\sigma: \mathbb{C} P^{1} \times \mathbb{C} P^{1} \rightarrow \mathbb{C} P^{1} \times \mathbb{C} P^{1}$ be an involution defined by the diagonal complex conjugation. Then the fixed point set of $\sigma$ is a torus and $\mathbb{C} P^{1} \times \mathbb{C} P^{1} / \sigma=S^{4}$. See Section 6 of [21] for this construction.

Let $X^{\prime}$ be a closed symplectic 4-manifold with $b_{2}^{+}\left(X^{\prime}\right)>1$. Now we take a connected sum $X=\mathbb{C} P^{1} \times \mathbb{C} P^{1} \sharp 2 X^{\prime}$ which is taken away from the branch set $T^{2}$. Then there is a double cover $X \rightarrow X^{\prime} \sharp S^{4}=X^{\prime}$ branched along $T^{2}$.

By the Seiberg-Witten vanishing theorem [22], there is no Seiberg-Witten basic class on $X$. Thus we have $\mathcal{S} W_{X}(\tilde{\xi})=0$ and $\mathcal{S} W_{X^{\prime}}(\xi)=k\left(X^{\prime}, T^{2}, a\right) \bmod 2$.

Since $X^{\prime}$ is a closed symplectic 4-manifold with $b_{2}^{+}\left(X^{\prime}\right)>1, \mathcal{S} W_{X^{\prime}}(\xi) \neq 0 \bmod 2$ for a Seiberg-Witten basic class $\xi$. Thus we have

$$
\mathcal{S} W_{X^{\prime}}(\xi)=k\left(X^{\prime}, T^{2}, a\right) \neq 0 \bmod 2 .
$$

\section{REFERENCES}

1. S. Akbulut, On quotients of complex surfaces under complex conjugation, J. Reine Angew. Math. 447 (1994), 83-90.

2. M. F. Atiyah and R. Bott, A Lefschetz fixed point formula for elliptic complexes: II. Applications, Ann. of Math. 88 (1968), 451-491. 1984).

3. W. Barth, C. Peters and A. Van de Ven, Compact complex surfaces (Springer-Verlag,

4. N. Bredon, Introduction to compact transformation groups (Academic Press, 1972).

5. Y. S. Cho, Finite group actions on the moduli space of self-dual connections I, Trans. Amer. Math. Soc. 323 (1991), 233-261. 
6. Y. S. Cho, Finite group actions on symplectic four-manifolds with finite fundamental groups, J. Austral. Math. Soc. Ser. A 66 (1999), 287-296.

7. Y. S. Cho, Finite group action and Gromov-Witten invariant, Preprint.

8. Y. S. Cho and Y. H. Hong, Cyclic group actions on 4-manifold, Acta. Math. Hung. 94 (4) (2002), 333-350.

9. S. K. Donaldson, The Seiberg-Witten equations and 4-manifold topology, Bull. Amer. Math. Soc. 33 (1996), 45-70.

10. S. K. Donaldson and P. B. Kronheimer, The geometry of 4-manifold (Clarendon Press, Oxford, 1990).

11. R. Fintushel and R. Stern, Pseudofree orbifolds, Ann. of Math. (2) 122 (1985), 335-364.

12. R. E. Gompf, A new construction of symplectic manifolds, Ann. of Math. (2) 142 (1995), 527-595.

13. R. E. Gompf and A. I. Stipsciz, 4-manifolds and Kirby calculus, Graduate Studies in Mathematics, Vol. 20, (Amer. Math. Soc., Providence, Rhode Island, 1999).

14. F. Hirzebruch, The signature of ramified coverings in Global analysis in honor of K. Kodaira (D. Spencer and S. Iyanage eds.) (Academic Press, 1969), 253-265.

15. R. Kirby, Problems in low-dimensional topology (Berkeley - 22 Dec 95).

16. P. B. Kronheimer and T. S. Mrowka, The genus of embedded surfaces in the projective plane, Math. Res. Letters 1 (1994), 797-808.

17. D. McDuff and D. Salamon, Introduction to symplectic topology (Clarendon Press, Oxford, 1995).

18. J. W. Morgan, T. S. Mrowka and Z. Szabó, Product formulas along $T^{3}$ for Seiberg-Witten invariants, Math. Res. Letters 4 (1997), 915-929.

19. J. W. Morgan, Z. Szabó and C. H. Taubes, A product formula for the Seiberg-Witten invariants and the generalized Thom conjecture, J. Differential Geom. 44 (1996), 706-788.

20. P. Ozsváth and Z. Szabo, The symplectic Thom conjecture, Ann. of Math. (2) 151 (2000), 93-124.

21. Y. Ruan and S. Wang, Seiberg-Witten invariants and double covers of 4-manifolds, Comm. Anal. Geom. 8 (2000), No. 3, 477-515.

22. D. Salamon, Spin geometry and Seiberg-Witten invariants (University of Warwick, October 2, 1995).

23. C. H. Taubes, The Seiberg-Witten invariants and symplectic forms, Math. Res. Letters 1 (1994), 809-822.

24. S. Wang, Gauge theory and involutions (Oxford University Thesis, 1990).

25. S. Wang, A vanishing theorem for Seiberg-Witten invariants, Math. Res. Letters 2 (1995), 305-310. 